\title{
Vagus nerve and phrenic nerve guided systematic nodal dissection for lung cancer
}

\author{
Zhenguo Liu ${ }^{1}$, Yao Liu ${ }^{1}$, Chunying Xie ${ }^{1}$, Jiali Yang ${ }^{1}$, Bo Zeng ${ }^{1}$, Sai-Ching Jim Yeung ${ }^{2}$, Chao Cheng ${ }^{1}$ \\ ${ }^{1}$ Department of Thoracic Surgery, The First Affiliated Hospital of Sun Yat-sen University, Guangzhou 510080, China; ${ }^{2}$ Department of Emergency \\ Medicine, The University of Texas MD Anderson Cancer Center, Houston, Texas, USA \\ Correspondence to: Chao Cheng, MD, PhD. Department of Thoracic Surgery, The First Affiliated Hospital of Sun Yat-sen University, 58 Zhongshan \\ 2nd Road, Guangzhou 510080, China. Email: drchengchao@163.com.
}

Submitted Jul 31, 2019. Accepted for publication Aug 12, 2019.

doi: $10.21037 /$ jtd.2019.08.80

View this article at: http://dx.doi.org/10.21037/jtd.2019.08.80

\section{Introduction}

Systematic nodal dissection (SND), which is defined as "all the mediastinal tissues containing the lymph nodes are dissected and removed systematically within anatomical landmarks", is vital in surgical treatment for patients with non-small cell lung cancer (NSCLC) with curative intent (1). However, for avoiding surgical complications or other reasons, a variation of SND affects the thoroughness of lymph node dissection and the accuracy of pathologic nodal (pN) staging, which are the most significant prognostic factors in resectable NSCLC $(2,3)$. Also, reduction in the severity and incidence of postoperative complications, such as nerve injury, is of utmost importance and remains challenging (4). Although some studies reported the experience and skills in mediastinal lymph node dissection $(4,5)$, a standard and systematic procedure for nerve protection are still lacking. Therefore, an easy-to-follow standardized approach of SND, which can not only achieve regional en bloc lymph node resection but also effectively protect the nerves, is critically needed.

Based on clinical practice experience, we discovered that the hilar and mediastinal lymph nodes are distributed along with the courses of these two nerves and connected in some anatomical regions (Figure 1). Thus, we first divided the lymph nodes into three areas of each side and developed an improved approach named "duo-nerve-guided SND” for resectable lung cancer, aiming to easily achieve complete resection of hilar and mediastinal lymph nodes as well as adipose tissue without increasing the nerve-related complications.

\section{Operative techniques}

From October 8, 2018, to January 31, 2019, 21 consecutive NSCLC patients, who presented with solid nodules and staged IA-IIIA by endobronchial ultrasonography (EBUS) and/or positron emission tomography/computed tomography (PET/CT) preoperatively and received surgery, were enrolled into this study. This study was approved by the Ethics Committee and Institutional Review Board of Guangdong Association Study of Thoracic Oncology (A2018006), and all patients signed the informed consent before this novel technique. Postoperative day and night cough were evaluated by the Cough Symptom Score, which scores cough severity from $0-5$ (mild to severe) (6). Atelectasis was diagnosed by X-ray or CT test after the operation. Diaphragmatic paralysis was detected by diaphragmatic ultrasound (7).

\section{Techniques}

We divide the hilar and mediastinal lymph nodes into three areas marked as A, B, C according to the course of the vagus and phrenic nerve and the distribution of lymph nodes (Figure 1A,B,C,D). Considering the anatomical differences between the left and right sides, we will introduce two sides, respectively.

\section{Part 1. For the left side (Figure 1A,B)}

The region inferior to the aortic arch is divided into two areas by the ligamentum arteriosum (areas A and B). (I) The posterior area of the left hilum (area A), is bordered 

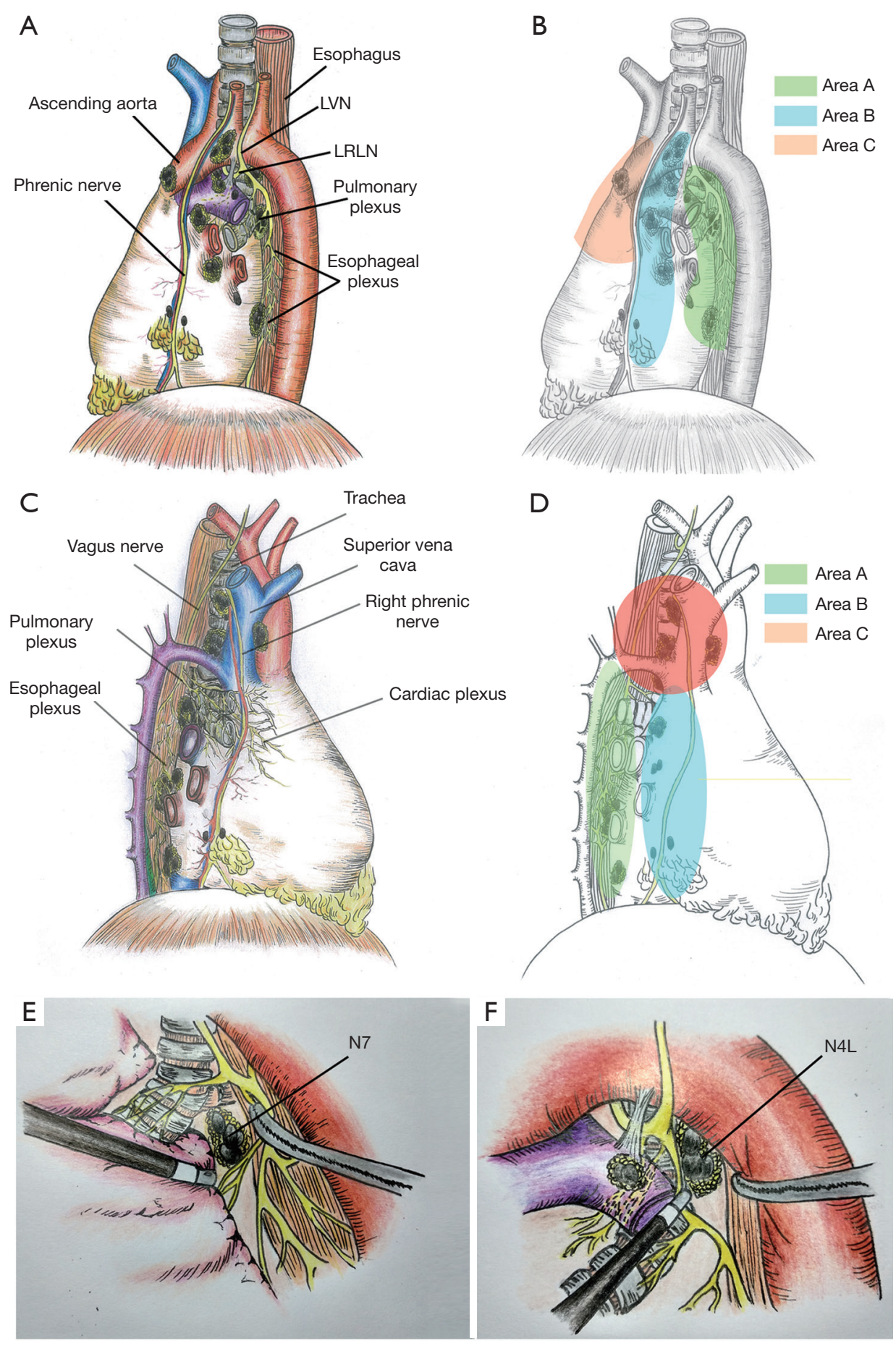

Figure 1 The anatomy of the thoracic vagus nerve and phrenic nerve and the distribution of lymph nodes. (A,C) The hilar and mediastinal lymph nodes were distributed along the vagus and phrenic nerves as well as their anatomical thoracic branches (pulmonary plexus, cardiac plexus, and esophageal plexus); (B,D) the regional lymph nodes distribution. Area A (green), the posterior area of the hilum; area B (blue), the anterior area of the hilum; area $\mathrm{C}$ (red), the anterior mediastinal area for the left side, the superior mediastinal area for the right side. (E) The subcarinal lymph nodes in left side (station 7); (F) the station 4L lymph nodes. RVN, right vagus nerve; LVN, left vagus nerve; LRLN, left recurrent laryngeal nerve. 
by ligamentum arteriosum, aortic arch, and descending aorta. This is where the left vagus nerve (LVN) successively divides into the left recurrent laryngeal nerve (LRLN), cardiac branch, posterior pulmonary branch, and esophageal plexus. Station 4L, 7, 8, 9, 10 lymph nodes are distributed in this area; (II) the anterior region of the left hilum (area B), is bordered by the phrenic nerve and the ligamentum arteriosum. Station 5, 6, and the anterior hilar lymph nodes (station 10) are in this area; (III) the anterior mediastinal region in front of the phrenic nerve (area $\mathrm{C}$ ), is bordered by the phrenic nerve and internal thoracic artery (ITA), and it contains the ascending aortic or anterior phrenic lymph nodes (station 6) and pericardial lymph nodes.

\section{Manipulation of left area A}

The mediastinal pleura was opened along the anterior edge of the descending aorta up to the posterior margin of ligamentum arteriosum, to expose the LVN trunk and its branches (Figure S1). The station 9, 8, 7, and 4L lymph nodes were dissected sequentially in the cephalad direction as guided by the vagus nerve.

A complete dissection of the station 7 lymph nodes from the left thoracic cavity is challenging, as the inferior area of the right main bronchus is challenging to expose (Figures 1E,2A). Based on our experience, when the left main bronchus is pushed anteriorly while the esophagus and LVN are retracted posteriorly by an assistant, the N7 lymph nodes can be exposed to allow en bloc removal. The LVN often sends out bronchial branches at the level of the carina into the left lower lung, and these branches often travel among the N7 nodes. Therefore, these branches must be carefully preserved during the radical resection of left upper lung cancer. However, the branches of LVN that predominantly innervate the left lower lung can be cut off first during radical resection of left lower lung cancer to facilitate station 7 lymph node dissection.

Station 4L lymph nodes dissection generally has a risk of LRLN injury because of anatomic limitations (Figures $1 F, 2 B)(8)$. To address this difficulty, the primary surgeon retracts the pulmonary artery and LVN anteriorly while an assistant retracts the esophagus posteriorly in order to expose the station $4 \mathrm{~L}$ nodes for resection.

\section{Manipulation of left area B}

The mediastinal pleura was opened along the posterior edge of the phrenic nerve from the level of the inferior pulmonary vein up to the para-aortic region. The LRLN is visualized, avoiding excessive dissociation or manipulation. Lymph nodes of stations 10, 5, 6, as well as the adipose tissue within this region, were dissected sequentially in the order above (Figure 2C,D).

\section{Manipulation of left area $\mathbf{C}$}

Using the phrenic nerve as the posterior border and the ITA as the anterior border, mediastinal lymph nodes and adipose tissue in this area was removed in a cephalad direction from the level of the aortic root to the level of superior edge of the aortic arch (Figure 2E,F).

\section{Part 2. For the right side (Figure 1C,D)}

(I) The posterior area of the right hilum (area A), where the right vagus nerve divides into pulmonary plexus and esophageal plexus below the azygos arch. Station 7, 8, 9, 10 lymph nodes are distributed in this area;

(II) The anterior area of the right hilum (area B), where the right phrenic nerve passes over the right side of the pericardium, and the station 10 and pericardial lymph nodes are distributed in this area;

(III) Superior mediastinal area (area C) is bordered by the vagus nerve, the phrenic nerve, and the azygos arch, where the station 2R, 4R lymph nodes are located.

\section{Manipulation of right area $A$}

We opened the mediastinal pleura inferiorly along the anterior edge of the esophagus up to the azygos arch to easily recognize and dissociate the vagus nerve trunk and its branches. Station 8, 9 lymph nodes are dissected firstly. To dissect station 7 lymph nodes, we carefully anatomized the pulmonary branches of the vagus nerve and lifted them to dissect the lymph nodes without transecting the pulmonary branches (Figure 3A,B).

\section{Manipulation of right area $B$}

The mediastinal pleura was opened along the course of phrenic nerve inferiorly to expose area B. The station 10 , pericardial lymph nodes as well as the adipose tissue, were dissected upwards, guided by the phrenic nerve (Figure 3C,D).

\section{Manipulation of right area $\mathbf{C}$}

Above the azygos arch, the mediastinal pleura was opened along the posterior edge of the superior vena cava and the anterior edge of the trachea, to firstly identify and expose the trunk of the right vagus nerve and its cardiac branches. Complete removal of station $4 \mathrm{R}$ and $2 \mathrm{R}$ lymph nodes together with adipose tissue was performed (Figure $3 E, F$ ).

In summary, mediastinal lymph nodes were dissected systematically along the course of the phrenic nerve by proceeding in the cephalad direction. The vagus nerve, phrenic nerve, and their branches were visualized throughout the procedure to protect them from being injured. 

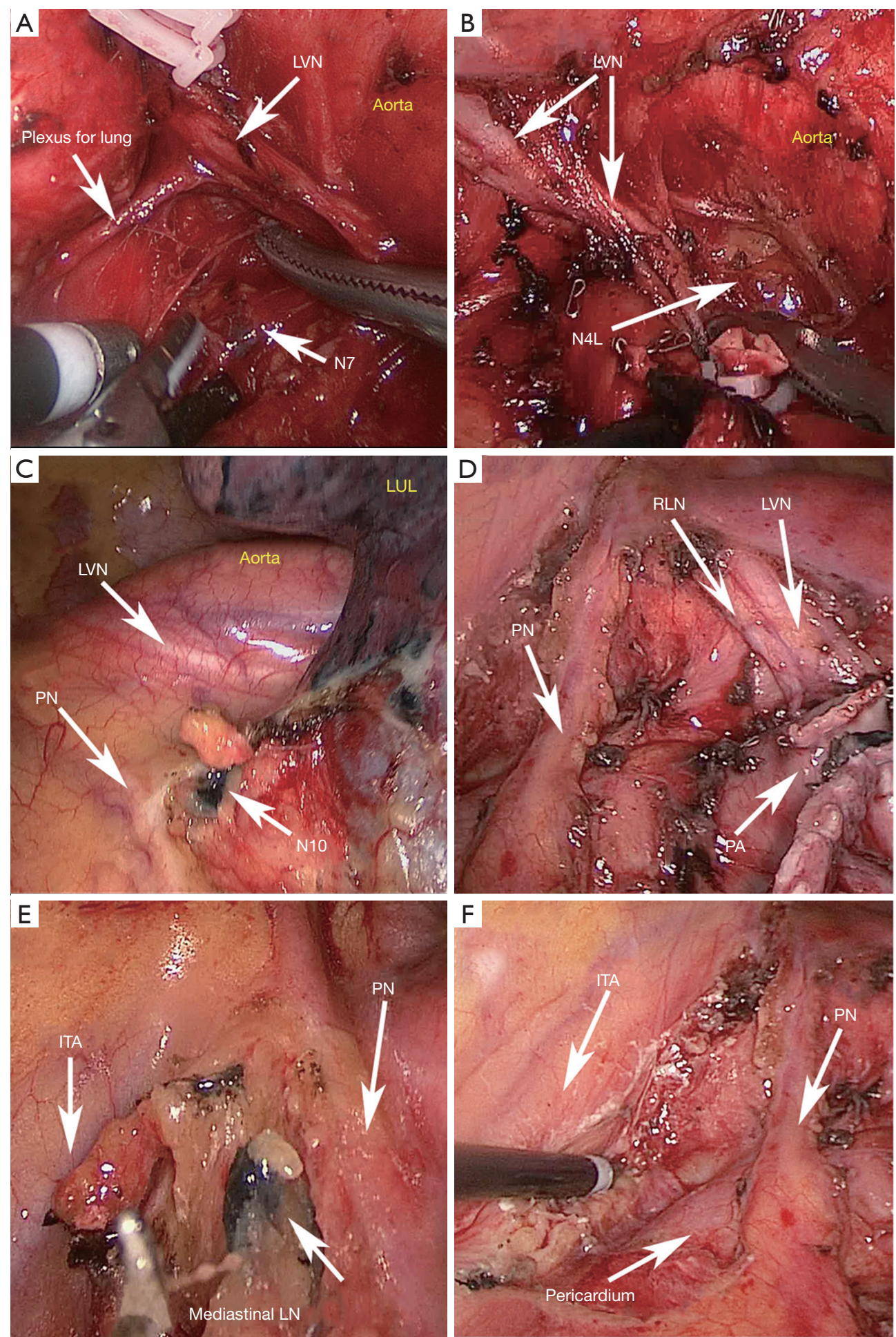

Figure 2 A representative surgical procedure in three areas of the left side. (A,B) The procedure of lymph nodes dissection in area A: (A) station 7; (B) station 4L; (C,D) lymph nodes dissection in area B; (E,F) lymph nodes and adipose dissection in area C. LUL, left upper lobe; LVN, left vagus nerve; PN, phrenic nerve; LRLN, left recurrent laryngeal nerve; ITA, internal thoracic artery; PA, pulmonary artery. 

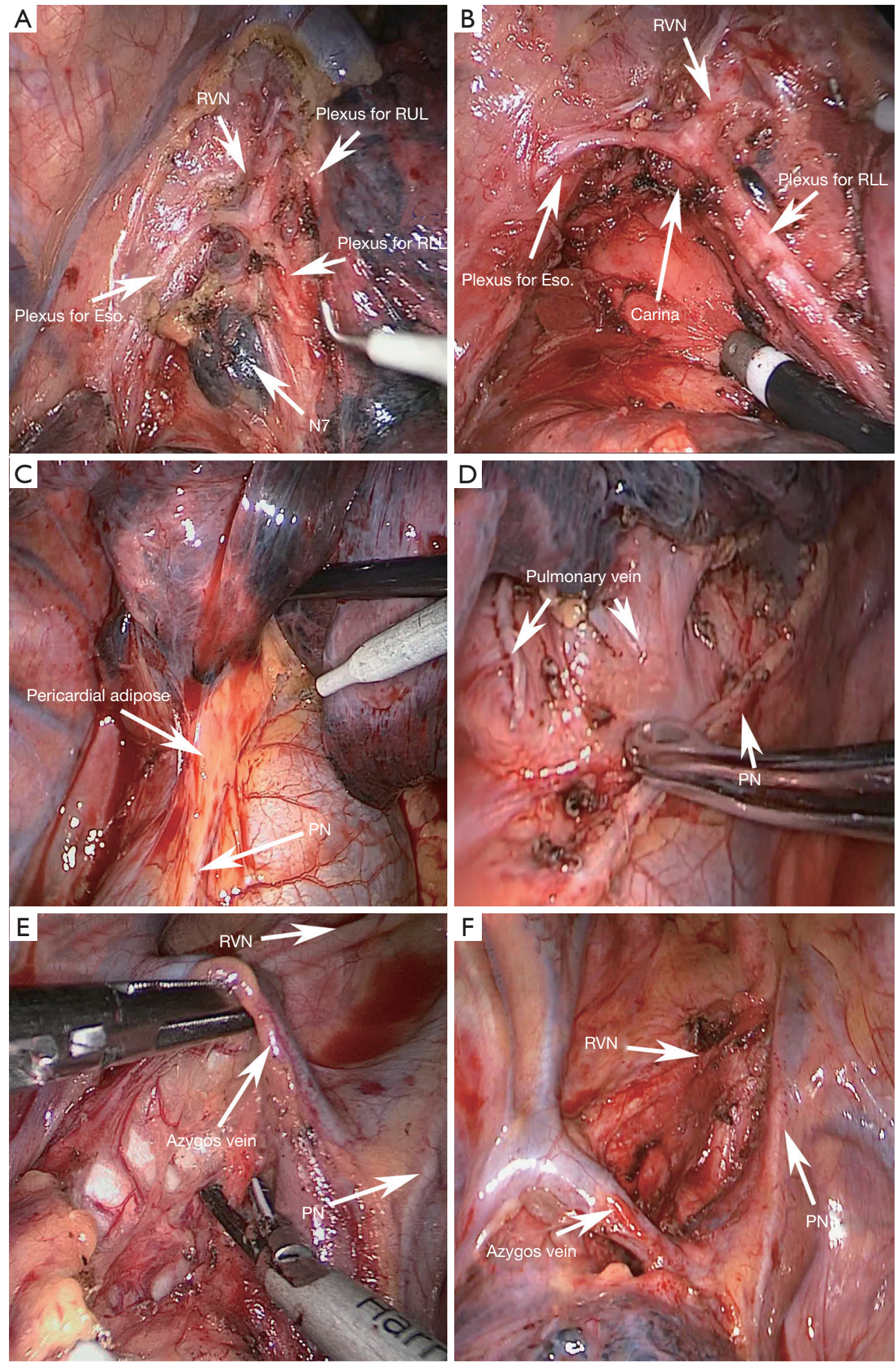

Figure 3 A representative surgical procedure in three areas of the right side. (A,B) The procedure of lymph nodes dissection in the right hilum area A: (A) station 7; (C,D) lymph nodes dissection in area B; (E,F) lymph nodes and adipose dissection in right area C. RUL, right upper lobe; RLL, right lower lobe; RVN, right vagus nerve; PN, phrenic nerve. 
Table 1 The clinical characteristics of 21 NSCLC patients

\begin{tabular}{lc}
\hline Patient characteristics & Number \\
\hline Age, mean [range] (years) & 57 [36-76] \\
Gender & 11 \\
Male & 10 \\
Female & \\
Histologic type & 17 \\
Adenocarcinoma & 2 \\
Squamous cell carcinoma & 2 \\
Others & \\
T status & 13 \\
T1 & 7 \\
T2 & 1 \\
T3 & 2 \\
Tumor location & 2 \\
Right upper lobe & 1 \\
Right middle lobe & 2 \\
Right lower lobe & \\
Left upper lobe* & \\
Left lower lobe & \\
\hline & \\
\hline &
\end{tabular}

*, one of the patients received left pneumonectomy. NSCLC, non-small cell lung cancer.

\section{Comments}

Considering that the incompleteness of lymph nodes dissection and the associated postoperative complications are two important concerns in the real world (3), we firstly proposed "duo-nerve-guided SND" for lung cancer based on our clinical experience. To better address the incompleteness of nodal dissection, we divided lymph nodes into three areas according to the anatomy of two nerves, and then en bloc removed the lymph nodes and adipose tissue in each area. We applied our strategy to 21 NSCLC patients (11 for right, 10 for left; and 11 males, 10 females) presenting with solid nodules. Patient characteristics were shown in Table 1. The mean duration of operation, blood loss, postoperative drainage (day 1) and postoperative hospital stay were $165.3 \pm 20.0 \mathrm{~min}, 55.4 \pm$ $25.4 \mathrm{~mL}, 177.9 \pm 66.2 \mathrm{~mL}$, and $4.0 \pm 1.4$ days, respectively. The mean number of dissected lymph nodes was $28.9 \pm 11.1$. Moreover, 7 of $21(33.3 \%)$ patients were confirmed to have up-regulated $\mathrm{pN}$ stage $(3 / 7 \mathrm{pN} 0-1,3 / 7 \mathrm{pN} 0-2,1 / 7$ pN1-2), comparing to $10.1 \%$ in previous report (9). Day and night cough were evaluated, and most of them had no cough or only mild cough (score $\leq 2$ ), only one patient had moderate night cough ( hoarseness or refractory cough (Table 2). Of note, no other severe postoperative complications were observed with a 2-week postoperative follow-up. The results indicate that duo-nerve-guided SND is a safe and feasible technique for complete lymph nodes dissection in the surgical treatment of lung cancer.

The intraoperative vagus nerve and phrenic nerve injuries, such as LRLN injury, increase postoperative complications and decrease quality of life $(4,10)$. In our study, we usually first dissociate and expose the nerves, then the nerves and their branches are visible and more easily protected. With this novel technique, none suffered from the refractory cough, hoarseness, or diaphragmatic paralysis (Table 2), suggesting the favorite results may be related to effective nerve preservation. In terms of operational skills, we would recommend that sharp dissection should be used whenever possible to remove the tissue near the recurrent laryngeal nerve instead of energetic instruments. In addition, one of our patients was found to have mediastinal lymph node metastasis in left area $\mathrm{C}$ while other lymph nodes were negative, upstaging from clinical N0 to pathological N2, which may reveal the necessity of dissection of the anterior mediastinal adipose tissue along with the lymph nodes (left area $\mathrm{C}$ and right area $\mathrm{B}$ ). Furthermore, using vagus nerve and phrenic nerve as anatomic landmarks and the concept of regional lymph node dissection (area A, B, and C), this improved technique is easy to follow and generalize.

Our favorable clinical outcomes of duo-nerve-guided SND suggest that it is an optimized and feasible approach for lung cancer. Further study based on a larger number of cases is being conducted at our center to evaluate the longterm clinical benefits of this surgical approach compared with current routine clinical practice (trial registration: ChiCTR1800018837, http://www.chictr.org.cn/). 
Table 2 TNM staging and postoperative complications of 21 NSCLC patients underwent duo-nerve guided SND

\begin{tabular}{|c|c|c|c|c|c|c|c|c|c|}
\hline $\begin{array}{l}\text { Preoperative } \\
\text { clinical stage }\end{array}$ & $\begin{array}{c}\text { Number } \\
\text { of } \\
\text { cases }\end{array}$ & $\begin{array}{c}\text { Postoperative } \\
\text { upstaged to } \\
\text { stage II (\%) }\end{array}$ & $\begin{array}{l}\text { Postoperative } \\
\text { upstaged to } \\
\text { stage III (\%) }\end{array}$ & $\begin{array}{l}\text { Upregulated } \\
\text { pN stage (\%) }\end{array}$ & $\begin{array}{c}\text { Number of } \\
\text { dissected } \\
\text { lymph nodes }\end{array}$ & \multicolumn{2}{|c|}{ Cough symptom ${ }^{*}$ (score $>2$ ) } & Hoarseness & $\begin{array}{c}\text { Diaphragmatic } \\
\text { paralysis }\end{array}$ \\
\hline II & 2 & 0 & 1 & 1 & $49,28^{\#}$ & 0 & 0 & 0 & 0 \\
\hline IIIA & 5 & 0 & 0 & 0 & $39.6 \pm 14.2$ & 0 & 0 & 0 & 0 \\
\hline
\end{tabular}

*, postoperative day and night cough was evaluated by the Cough Symptom Score, which scores cough severity from 0-5 (mild to severe);

\#, the number of dissected lymph nodes in these two patients was 49 and 28, respectively. NSCLC, non-small cell lung cancer; SND, systematic nodal dissection.

\section{Acknowledgments}

Funding: This work was supported by grants from China National Natural Sciences Foundation (No.81572391 to C Cheng), Science and Technology Planning Project of Guangdong Province Foundation (No. 2016A020215050 and 2017A020215167 to C Cheng).

\section{Footnote}

Conflicts of Interest: The authors have no conflicts of interest to declare.

Ethical Statement: The authors are accountable for all aspects of the work in ensuring that questions related to the accuracy or integrity of any part of the work are appropriately investigated and resolved. Written informed consent was obtained from the patients for publication of this manuscript and any accompanying images.

\section{References}

1. Lardinois D, De Leyn P, Van Schil P, et al. ESTS guidelines for intraoperative lymph node staging in non-small cell lung cancer. Eur J Cardiothorac Surg 2006;30:787-92.

2. Smeltzer MP, Faris NR, Ray MA, et al. Association of Pathologic Nodal Staging Quality With Survival Among Patients With Non-Small Cell Lung Cancer After Resection With Curative Intent. JAMA Oncol 2018;4:80-7.

3. Verhagen AF, Schoenmakers MC, Barendregt W, et al. Completeness of lung cancer surgery: is mediastinal dissection common practice? Eur J Cardiothorac Surg 2012;41:834-8.

4. Watanabe A, Nakazawa J, Miyajima M, et al. Thoracoscopic mediastinal lymph node dissection for lung cancer. Semin Thorac Cardiovasc Surg 2012;24:68-73.

5. Li XD, Yang H, Zheng YB, et al. Video-assisted thoracoscopic surgery (VATS) right upper lobectomy and systematic lymph node dissection for lung cancer. J Thorac Dis 2013;5 Suppl 3:S289-90.

6. Hsu JY, Stone RA, Logan-Sinclair RB, et al. Coughing frequency in patients with persistent cough: assessment using a 24 hour ambulatory recorder. Eur Respir J 1994;7:1246-53.

7. Mandoorah S, Mead T. Phrenic Nerve Injury. Treasure Island, FL: StatPearls, 2019.

8. Witte B, Hürtgen M. Video-assisted mediastinoscopic lymphadenectomy. Multimed Man Cardiothorac Surg 2007;2007:mmcts.2006.002576.

9. Wilson JL, Louie BE, Cerfolio RJ, et al. The prevalence of nodal upstaging during robotic lung resection in early stage non-small cell lung cancer. Ann Thorac Surg 2014;97:1901-6; discussion 1906-7.

10. Huang J, Luo Q, Tan Q, et al. Evaluation of the surgical fat-filling procedure in the treatment of refractory cough after systematic mediastinal lymphadenectomy in patients with right lung cancer. J Surg Res 2014;187:490-5.

Cite this article as: Liu Z, Liu Y, Xie C, Yang J, Zeng B, Yeung SC, Cheng C. Vagus nerve and phrenic nerve guided systematic nodal dissection for lung cancer. J Thorac Dis 2019;11(9):40214027. doi: $10.21037 /$ jtd.2019.08.80 


\section{Supplementary}

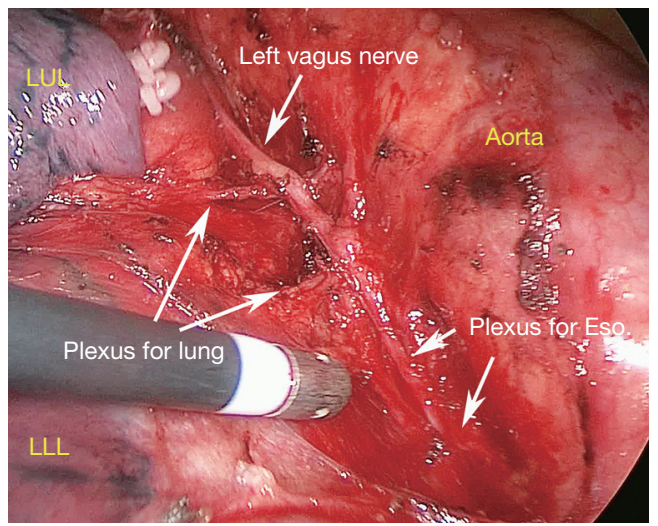

Figure S1 A representative surgical procedure to expose the left vagus nerve trunk and its branches. LUL, left upper lobe; LLL, left lower lobe; Eso, esophagus. 